\title{
LA GUILLOTINE PROJETÉE SUR SCÈNE: LES LIAISONS DANGEREUSES (1982) DE LACLOS REVISITÉES PAR CHRISTOPHER HAMPTON DANS L'ANGLETERRE DE MARGARETH THATCHER
}

Maristela Gonçalves Sousa Machado ${ }^{1}$

Résumé: La relecture théâtrale des Liaisons dangereuses (1782) de Choderlos de Laclos signée par Christopher Hampton acquiert une signification particulière dans le contexte historique de la Grande-Bretagne de la première ministre Margareth Thatcher dans les années 1980. Le dramaturge anglais prend position face à un libéralisme économique, qui a provoqué de fortes tensions sociales, en opérant un déplacement hardi dans le roman. II y met en relief un autre type de punition infligé à Madame de Merteuil non pas comme conséquence du conflit des vanités de libertins et des disputes de pouvoir, mais par les circonstances d'un moment historique.

Mots-clés: Les Liaisons dangereuses (1782), adaptation théâtrale, Christopher Hampton.

Resumo: A releitura teatral de As relações perigosas (1782) de autoria de Christopher Hampton assume um significado específico no contexto histórico da Grã-Bretanha dos anos 1980. O dramaturgo inglês toma posição diante da política de um liberalismo econômico, que provocou fortes tensões sociais, ao operar uma ousada modificação na intriga do romance: trata-se de uma punição infligida ao personagem de Madame de Merteuil, que não resulta dos conflitos de vaidade e da disputa de poder entre os libertinos, mas pelas circunstâncias de um momento histórico.

Palavras-chave: As relações perigosas (1782), adaptação teatral, Christopher Hampton.

Comme devant tant d'œuvres de notre temps - pas seulement littéraires - le lecteur des Liaisons eût pu dire: 'Ça ne peut durer ainsi'.

André Malraux

\section{Introdução}

Les Liaisons dangereuses de Choderlos de Laclos, roman épistolaire polyphonique du XVIII ${ }^{e}$ siècle, se présente comme un recueil de lettres et aborde les rapports de pouvoir entre la marquise de Merteuil et le vicomte de Valmont, deux aristocrates libertins, séduisants, aux mœurs corrompues qui trament des intrigues contre des victimes innocentes et se moquent de l'amour. II s'agit d'une œuvre qui encore aujourd'hui demeure ouverte à des lectures multiples comme l'attestent plusieurs relectures critiques

\footnotetext{
${ }^{1}$ Professora Adjunta do Centro de Letras e Comunicação da Universidade Federal de Pelotas (UFPEL).
}

Revista Eletrônica Literatura e Autoritarismo, no 21- Janeiro-Junho de 2013 - ISSN 1679-849X $\mid 110$ http://cascavel.ufsm.br/revistas/ojs-2.2.2/index.php/LA/about/index 


\section{Literatura e Autoritarismo}

\section{Identidade, memória e representações culturais}

contemporaines: romans, pièces de théâtre, films, opéras, ballets et séries télévisuelles actualisent le roman avec une fréquence et une variété remarquables. Des Liaisons dangereuses (Virgin, 1998) de Bruno Beausir, alias Doc Gynéco, rappeur français hargneux dont ce deuxième disque est ainsi baptisé "par connivence avec le Valmont de Laclos" (GRASSIN, 1999, s/p) à la relecture cinématographique du cinéaste chinois Jin-ho Hur lancée en 2012, le cortège de recréations est interminable.

Que dire de la présence du roman de Laclos au Brésil? Carlos Drummond de Andrade l'a traduit à la perfection en 1947. Drummond ne le fait pas sur commande de l'Editora Globo, responsable de la publication, mais par le défi de travailler avec une œuvre qu'il a placée sur sa liste des dix meilleurs romans. Le poète écrit une préface du traducteur où il manifeste son enthousiasme pour "um dos mais extraordinários retratos da alma humana dirigida para um só fim terrestre, com que a literatura já tenha assustado nossa ignorância de nós mesmos" (DRUMMOND, s/d, p. 15). Osório Borba (1947), Sérgio Milliet (1961) et Fernando Cacciatore de Garcia (2008) ont également traduit le roman au Brésil. Dorothée de Bruchard signe en 2012 la traduction brésilienne la plus récente des Liaisons dangereuses et la critique souligne son actualité et sa vigueur (Fierro, 2012).

Dans ce travail, nous avons choisi d'étudier une des actualisations du roman de Choderlos de Laclos, Les Liaisons dangereuses (1985), réécriture théâtrale de Christopher Hampton. Né en 1946 à Fayal aux Açores, Hampton est un des auteurs dramatiques vivants les plus influents de la Grande-Bretagne. Après une licence de français et d'allemand avec un First Class Honours Degreee au New College à Oxford, il travaille comme resident dramatist au Royal Court Theatre à Londres et devient l'auteur le plus jeune à avoir une pièce - When did you last see my mother? - jouée à Londres en 1966. Pendant les années 1970, il continue à écrire pour le théâtre, produit un nombre de pièces réussies - Total eclipse, The philanthropist, Treats et savages - toujours au Royal Court Theatre et évolue à contre-courant d'une époque dominée par les expériences avec le théâtre non verbal et par la critique acérée de Somerset Maugham, George Bernard Shaw, et Noel Coward. Ignorant les débats des magazines intellectuels sur la mort du langage au théâtre, Hampton s'inspire du Misanthrope de Molière pour écrire The philanthropist, fréquente la comedy of manners, et se sert des répliques bien envoyées tout en abordant les thèmes de l'actualité tels que l'aliénation, les limites du langage, les privilèges de l'académie. 
Hampton traduit des classiques comme Oncle Vanya de Tchekhov, Hedda Gabler et $A$ doll's house d'lbsen, ainsi que des pièces contemporaines comme Art de Yasmina Reza. Il adapte Tartuffe (1983) de Molière, Les Liaisons dangereuses (1985) de Laclos et Sunset Boulevard (1995) de Billy Wilder qui, acclamées par la critique et par le public, ont consolidé sa carrière au théâtre. II écrit aussi pour la télévision et pour le cinéma. Avec le scénario de Dangerous liaisons (1988), film de Stephen Frears, il a gagné pour le meilleur scénario adapté l'Oscar et Writers Guild of America en 1989, le BAFTA (British Academy of Film and Television Arts) et le London Critics Circle Film Award, en 1990.

Il est évident qu'il s'agit d'un auteur qui porte un intérêt considérable à la littérature, la relecture de romans et de pièces théâtrales aux auteurs les plus divers et aux thèmes variés. Pourquoi Christopher Hampton a-t-il choisi Laclos? II l'explicite après la didascalie initiale du texte publié de ses Liaisons dangereuses (1985) "A Note on Laclos". II y présente Laclos avec un enthousiasme non caché, trace les lignes générales de sa biographie et conclut que le seul roman écrit par Laclos - "not merely a masterpiece, but the supreme example of its genre", "his vigorously classical novel", "this extraordinary and meteoric work" - demeure une énigme que la biographie de l'auteur n'éclaire qu'avec "an intriguing light" (LLH-H, p.7). ${ }^{2}$

Pourquoi Christopher Hampton a-t-il choisi de produire une version théâtrale des Liaisons dangereuses? Les attentes du lecteur avisé sont orientées par cette "Note on Laclos": il s'agit du regard d'un universitaire, licencié en littérature française à Oxford, mais aussi d'un homme de théâtre expérimenté, qui aime confronter les classiques de la littérature comme on l'a vu dans sa production pour le théâtre, sur un roman qu'il admire profondément. Mais il ne s'agit pas de la première relecture théâtrale du roman.

\section{Les Liaisons dangereuses au théâtre}

Le parcours du récit romanesque à la scène suscite aussi un intérêt particulier quand il s'agit de la relecture d'un roman épistolaire du XVIII ${ }^{\mathrm{e}}$ siècle - le théâtre était le divertissement préféré de l'époque - imprégné de références théâtrales (MACHADO,

\footnotetext{
2 L'indication LLD-H suivie d'un numéro de page renvoie à HAMPTON, Christopher. Les liaisons dangereuses. London: Faber and Faber, 1986.
}

Revista Eletrônica Literatura e Autoritarismo, no 21- Janeiro-Junho de 2013 - ISSN 1679-849X $\mid 112$ http://cascavel.ufsm.br/revistas/ojs-2.2.2/index.php/LA/about/index 
2005). Les descriptions de décors, d'ambiance et de personnages y sont moins importantes que le rapport entre les personnages et le dialogue épistolaire. L'échange de lettres fait souvent penser à l'échange de répliques. En outre il y a des références récurrentes à des pièces et à des dramaturges, le caractère tragique d'une action condensée dans le temps et dans l'espace et le véritable souci de mise en scène que manifestent Valmont et Merteuil. Pour nous, le roman se présente souvent sous une perspective relative à la mise en scène et au jeu de l'acteur, la notion de jeu - qu'il s'agisse de compétition, de hasard, de simulacre ou de vertige - étant envisagée sur le plan du comportement, de la représentation sociale, de la dramaturgie ou de l'écriture même. La tension et le mouvement imprimés par l'échange de lettres semblent projeter le texte vers une scène toujours à venir. En effet, l'absence de narrateur, le caractère fragmentaire des discours, le déplacement des points de vue, les ruptures du rythme et l'entrecroisement des lignes narratives propres au roman épistolaire polyphonique contribuent à l'engendrement d'un récit qui peut se prêter à un découpage semblable aux actes, scènes et tableaux d'une pièce théâtrale.

Michel Delon (1987) raconte que la première adaptation théâtrale du roman de Laclos date de 1783, Le danger des liaisons de Beaunoir. Puis Ancelot et Saintine transforment le roman en drame bourgeois en 1834; Lucien Gumpel et Georges Delaquys créent Monsieur de Prévan ou le législateur de Cythére en 1907; la même année, Pierre Nozière écrit ses Liaisons dangereuses dans le ton du théâtre de boulevard. Paul Achard crée un montage présenté au théâtre Montparnasse-Gaston Baty en 1952. Laurent Versini (1987) trace également un historique des adaptations théâtrales des Liaisons dangereuses. Plus récemment Quartett d'après Laclos (1982) ou tout simplement Quartett (comme la pièce est devenue connue) de Heiner Müller - dont la première mise en scène en France a été dirigée par Patrice Chéreau au théâtre des Amandiers de Nanterre en 1985 - a suscité un grand nombre de critiques en fonction des transformations hardies proposées par le dramaturge berlinois. Philippe Faure en a proposé une adaptation en 2001 et en 2003, Giles Havergal a composé une relecture commandée par le prestigieux American Conservatory Theater de San Francisco.

Il est vrai que de nouvelles incarnations du roman de Laclos se succèdent et il est courant d'entendre dire à propos des adaptations théâtrales que, au bout de dix ans, elles deviennent datées. La pièce de Christopher Hampton semble montrer le contraire: dans 
les commentaires concernant toutes les adaptions postérieures à sa création, elle est toujours citée comme exemple de travail réussi, espèce de référence idéale qu'il faut poursuivre. "A brief overview of critical reactions to Hampton's Les Liaisons dangereuses reveals almost universal admiration for the dramatic adaptation of Laclos' 18th Century novel", affirme Stephanie Barbé Hammer (1990, p.109) qui cite plusieurs articles des principaux quotidiens londoniens dans un des chapitres du volume qui réunit les essais de différents auteurs sur la production théâtrale de Christopher Hampton (GROSS, 1990).

Comme nous l'avons déjà remarqué, après la didascalie initiale du texte publié de ses Liaisons dangereuses (1985), Hampton écrit "A Note on Laclos" où il présente l'auteur avec un enthousiasme non caché, trace les lignes générales de sa biographie et conclut:

Geometrician, inventor, military strategist, feminist, revolutionary, devoted husband and father: all of these qualities, some initially surprising in the author of Les Liaisons dangereuses, other less so, make their contribution towards a way of looking at this extraordinary and meteoric work; without, however, exhausting the pleasures of its mystery and merciless intelligence. (LLD-H, p.7)

Hampton ne parle que de Laclos et de son roman dans cette note introductrice. Pas un mot à propos de son propre texte, de sa relecture, de ses motivations. "A note on Laclos" suggère d'ailleurs une attitude assez respectueuse, une grande admiration pour l'œuvre de l'auteur français. C'est donc un intérêt personnel et une persistance considérable qui motivent sa dramatisation des Liaisons dangereuses pour la Royal Shakespeare Company.

L'adaptation théâtrale des Liaisons dangereuses de Hampton, jouée pour la première fois en janvier 1985 à Stratford-Upon-Avon, a eu beaucoup de succès; le même retentissement se répète à l'Ambassador Theatre à Londres qui donne la pièce pendant cinq ans et demi, à Broadway et dans plusieurs pays dont la France. La RSC a présenté la version anglaise des Liaisons dangereuses (1985) au théâtre Renaud-Barrault à Paris en 1990 et une adaptation de celle-ci en français, signée par Jean-Claude Brisville, a été jouée pour la première fois en 1986 au théâtre Édouard VII, avec Bernard Giraudeau et Caroline Cellier dans les rôles principaux.

L'opinion des spécialistes de l'œuvre de Laclos est un peu différente. Michel Delon n'est pas élogieux à l'égard de l'adaptation de Hampton - pour lui le dramaturge voulait 


\section{Literatura e Autoritarismo}

"rendre un certain libertinage à la française" - et arrive même à déplorer sa publication et représentation en France: "On peut regretter que cette adaptation anglaise ait été retraduite en français sinon dans la langue de Laclos par Jean-Claude Brisville (Actes Sud, 1988) et montée à Paris avec Caroline Cellier et Bernard Giraudeau" (1999, p. 113).

Pour Laurent Versini, en dépit ce certaines inadéquations de gestes et de langage dans la traduction française de Jean-Claude Brisville et d'une méconnaissance de "la thématique et de la stylisation de la tradition française" qu'il ne spécifie pas -, la pièce est "un centon du roman" et Christopher Hampton un "très bon connaisseur du dix-huitième siècle français formé à l'université de Cambridge [sic]“ qui "n’ignore pas la technique de la contraction de texte“ (1993, p. 212).

Maintenant que nous avons une idée de la réception des Liaisons dangereuses (1985), nous allons passer au texte de Hampton sans aucune intention d'en réaliser une étude exhaustive. Nous n'avons pas non plus l'intention de faire un bilan des modifications, suppressions, resserrements et ajouts par rapport au roman. Tâchons donc de regarder de plus près les tableaux que le dramaturge Christopher Hampton a créés et d'y chercher les traces de théâtralité comme on l'a fait pour le roman de Laclos.

En 2012, John Malkovich, l'acteur qui a joué le rôle de Valmont dans le film de Stephan Frears, revisite l'œuvre de Hampton au théâtre de l'Atelier à Paris mettant en scène de jeunes acteurs qui, portables et tablettes électroniques à la main, parlent la langue française du XVIIle siècle. Plus de vingt cinq ans après sa première présentation, la version théâtrale de Hampton provoque encore l'admiration du public et de la critique.

Au Brésil, Mauro Batista Vedia a signé la mise en scène originale de As ligações perigosas à São Paulo en 2010, avec adaptation et traduction de Clara Carvalho et Rachel Ripani présentant Maria Fernanda Cândido et Marat Descartes dans les rôles de Merteuil et de Valmont.

Mais revenons au texte publié de la pièce de Hampton.

\section{De la réecriture}




\section{Literatura e Autoritarismo}

Identidade, memória e representações culturais

Christopher Hampton a transposé Les liaisons dangereuses en une pièce en deux actes contenant chacun neuf tableaux. Selon la didascalie initiale, l'action se situe "in various salons and bedrooms in a number of hotels and châteaux in and around Paris, and in the Bois de Vincennes, one autumn and winter in the 1780s." Après les indications de l'espace scénique et du temps, s'ensuivent les noms des neuf personnages principaux marquise de Merteuil, $\mathrm{M}^{\text {me }}$ de Volanges, Cécile de Volanges, vicomte de Valmont, Azolan, $\mathrm{M}^{\mathrm{me}}$ de Rosemonde, présidente de Tourvel, Émilie, chevalier Danceny et la référence à "various servants in the Merteuil, Rosemonde, Tourvel and Valmont households" (LLD-H, p. 6). Les noms des personnages sont accompagnés des noms des acteurs qui ont participé à la première à The Other Place, Statford-upon-Avon, le 24 septembre 1985. En plus apparaissent: Director: Howard Davies et Designer: Bob Crowley.

Avant même de commencer la lecture des dialogues, le lecteur découvre l'emploi de plusieurs termes en langue française (marquise, Madame, vicomte, présidente, chevalier, hôtels, châteaux, Opéra, Bois de Boulogne). Le dramaturge situe l'intrigue "in

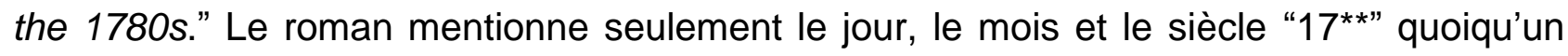
certain nombre d'indications de l'intrigue permette de situer l'action vers les années 1760.

Certaines traces du regard anglais de Christopher Hampton sur Les Liaisons dangereuses de Laclos portent la marque de l'ironie. C'est le cas de l'inévitable thé de cinq heures - "A September afternoon. VALMONT is taking tea with LA MARQUISE DE MERTEUIL in her grand salon" (LLD-H, p. 29) -, de la figure d'un majordome (LLD-H, p. 9$10,33-34,36,38,76)$ et du commentaire sur la pluie. II nous semble évident que le choix de Hampton n'est pas gratuit: ce sont des éléments - le thé, la pluie, le majordome populairement reconnus comme emblématiques de la culture anglaise. Mais il y a certainement plus. Certains tableaux des Liaisons dangereuses de Hampton sont caractérisés par une grande mobilité des personnages qui leur donne un caractère fragmentaire. Ce va-et-vient évoque un genre très connu dans le théâtre anglais: the comedy of manners avec très peu d'action, une intrigue artificielle et chargée de dialogues cérébraux, cyniques et pleins d'esprit - le fameux wit anglais. Genre qui a eu son apogée pendant la période de la Restauration (1660-1685) et était essentiellement fondé sur la satire des mœurs, de l'hypocrisie et du matérialisme chez les aristocrates.

\footnotetext{
${ }^{3}$ Nous avons fait la citation de toutes les didascalies de la pièce en italique.
}

Revista Eletrônica Literatura e Autoritarismo, no 21- Janeiro-Junho de 2013 - ISSN 1679-849X |116 http://cascavel.ufsm.br/revistas/ojs-2.2.2/index.php/LA/about/index 


\section{Literatura e Autoritarismo}

Identidade, memória e representações culturais

Revenons à l'intrigue de la pièce: au premier tableau de l'acte premier des Liaisons dangereuses de Hampton, comme dans une scène d'exposition classique, le lecteur est présenté aux principaux personnages de la trame ainsi qu'aux motifs qui la déclenchent. À part l'absence de verbes dans certaines phrases, une longue indication scénique au début de ce premier tableau fait penser à une description romanesque. Voyons: l'action commence pendant une chaude soirée au mois d'août dans le somptueux salon principal ("suggestions of great opulence") de l'hôtel de la marquise de Merteuil à Paris. Elle joue au piquet avec $\mathrm{M}^{\mathrm{me}}$ de Volanges qui est assise à côté de sa fille Cécile ("a slim and attractive blonde girl of 15") qui semble s'ennuyer et avoir sommeil ("politely stifling the occasional yawn"). Merteuil interrompt le silence - on a l'impression que les dames sont concentrées sur le jeu, il n'y a que le claquement des cartes qui est audible ("the large playing cards slap down on one another", LLD-H, p. 9) - et la conversation commence.

Elles parlent de Cécile, qui vient de sortir du couvent et commence à avoir des expériences dans "the outside world" (LLD-H, p. 9). Le majordome arrive et annonce discrètement la présence de monsieur le vicomte de Valmont. Avant qu'il arrive, les deux femmes parlent de sa mauvaise réputation et du fait que celle-ci ne l'empêche pas d'être reçu par la bonne société. Un dialogue cordial s'établit mais, après la sortie de la mère et des la fille, Merteuil et Valmont adoptent un autre ton.

Pour se venger de monsieur de Gercourt, un ancien amant, la Marquise demande au Vicomte (lui aussi un ancien amant) de corrompre la jeune Cécile de Volanges, l'invitée qui vient de sortir, promise à Gercourt. Valmont refuse à cause d'un autre projet: il va partir à la campagne, au château de $M^{\text {me }}$ de Rosemonde, sa vieille tante, dont il veut séduire l'hôte - la vertueuse présidente de Tourvel. Le point de départ de l'histoire est donc exactement le que celui du roman.

Après ces moments initiaux, l'intrigue se déroule: pour se venger de $M^{\text {me }}$ de Volanges - elle a prévenu la Présidente contre lui -, le Vicomte change d'idée et décide de participer au projet de la Marquise. Cécile et le timide Chevalier Danceny tombent amoureux. Les deux libertins feignent de les aider et deviennent leurs confidents. Valmont séduit Cécile et devient son amant. À la fin du dernier tableau du premier acte, $\mathrm{M}^{\mathrm{me}}$ de Rosemonde conseille à la Présidente qui vient de lui avouer son amour pour le Vicomte: "If he has let you go, you must go." (LLD-H, p. 65). Les recommandations sont inutiles. 
Recourant à des ruses habiles avec l'aide de son chasseur Azolan et même du confesseur de Tourvel - Father Anselme, "an amiably dim-witted Cistercian" (LLD-H, p. 76) -, le libertin finit par la conquérir tout en s'éprenant d'elle. Jalouse, Merteuil se brouille avec son complice et le défie de rompre brutalement avec la Présidente, qui tombe malade. À son tour, Valmont frustre les plans de la Marquise qui voulait prendre Danceny comme amant.

C'est la guerre entre les libertins. La libertine incite le jeune homme à se battre en duel avec Valmont qui meurt après avoir avoué à Danceny son amour pour la Présidente. Au dernier tableau de la pièce, un changement significatif par rapport á la fin du roman: mesdames de Volanges, de Rosemonde et de Merteuil jouent aux cartes dans le salon de la Marquise. Le lecteur apprend que la Présidente est morte au couvent aussitôt après avoir reçu la nouvelle de la mort de Valmont. Le Chevalier s'enfuit à Malte. $\mathrm{M}^{\text {me }}$ de Volanges ne comprend pas pourquoi Cécile a décidé d'entrer au couvent. De façon énigmatique, $\mathrm{M}^{\text {me }}$ de Rosemonde raconte qu'elle a reçu "a very strange letter from Danceny" (LLD-H, p.100) et suggère, sans donner d'explications, que la décision de la jeune fille doit être respectée.

Dans la dernière réplique de la pièce, Merteuil exprime des propos optimistes sur le futur: "I dare say we would not be wrong to look forward to whatever the nineties may bring." Les trois personnages reprennent leur jeu de cartes. C'est un signe visuel, une image brève mais nette qui termine la pièce - "The atmosphere is serene. Very slowly, the lights fade, but just before they vanish, there appears on the wall, fleeting but sharp, the unmistakable silhouette of the guillotine" (LLD-H, p.10).

Du point de vue du développement de l'intrigue, on peut donc remarquer que cette adaptation ne présente aucune modification significative, elle a essentiellement gardé l'action du roman de Laclos et même repris plusieurs discours des personnages et plusieurs situations. II est évident que certains épisodes et personnages secondaires du roman de 379 pages ont été éliminés pour accélérer l'allure du récit, mais c'est à la fin de la pièce qu'émerge un élément radicalement nouveau et différent: la destinée finale de Merteuil. 


\section{Literatura e Autoritarismo}

Identidade, memória e representações culturais

\section{Une relecture politique}

L'édition des Liaisons dangereuses de Christopher Hampton porte en épigraphe un commentaire emprunté à André Malraux: "Comme devant tant d'œuvres de notre temps pas seulement littéraires - le lecteur des Liaisons eût pu dire: 'Ça ne peut pas durer ainsi'." Ce choix peut suggérer d'emblée un côté politique dans la relecture d'une œuvre qui a précédé de quelques années la Révolution française de 1789. D'ailleurs, le choix des années 1780 comme repère temporel (différemment, comme nous l'avons déjà dit, de l'intrigue du roman qui situe l'action en $17^{* *}$ ) posé explicitement au début de la pièce, semble confirmer cette option.

En effet, il y a, chez la majorité des critiques anglais, le sentiment que la pièce de Hampton est un produit des années 1980 et traduit l'esprit de l'époque. En GrandeBretagne, c'était le gouvernement de Margaret Thatcher qui avait une forte identification avec l'administration Ronald Reagan aux États-Unis. II s'agit évidemment d'une question complexe, mais la discussion sur le libéralisme qui touche profondément la GrandeBretagne dans les années 1980 - "a sort of sanctification of institutionalized selfishness", selon Christopher Hampton (MACCUBIN, 1990, p. 82) trouve peut-être des échos dans une des polémiques qui dominent le XVIIle siècle du point de vue de l'économie politique: depuis Bernard Mandeville jusqu'à Adam Smith en passant par les physiocrates, et avec des variations selon chaque théoricien, on prêchait une diminution du contrôle des gouvernements et une augmentation des libertés individuelles comme condition nécessaire à la prospérité des pays. Pour les critiques de ces idées, la liberté individuelle donnait libre cours à l'exercice de la cupidité, de l'avidité.

C'est peut-être le bon moment pour faire une brève parenthèse et commenter certains aspects politiques des Liaisons dangereuses, le roman. Pour certains auteurs, le roman est un signe précurseur de la Révolution de 1789. Baudelaire (1968, p. 644) par exemple, y reconnaît l'énergie satanique du mal qui allait détruire l'ancien ordre social "La Révolution a été faite par des voluptueux [...]. Les livres libertins commentent donc et expliquent la Révolution." René Pomeau conteste la vision de ceux qui ont vu dans le roman "les prémices de la révolution politique et sociale qu'avec le recul nous voyons poindre à l'horizon en 1782" (1981, p. 58): 
Laclos sans doute pas plus que ses contemporains ne devinait le tour qu'allaient prendre les événements. Mais il partage leur état d'esprit prérévolutionnaire, et son roman en porte la marque. On se gardera pourtant d'appliquer ici des schémas adaptés aux sociétés industrielles des $X I X^{e}$ et $X X^{e}$ siècles, dont il ne pouvait avoir aucune idée. (POMEAU, p. 60).

Effectivement, le roman peut être lu dans une perspective politique si l'on considère le portrait des aristocrates désœuvrés qui passent leur temps à jouer aux cartes, à aller à la chasse, à potiner ou à tisser des toiles pour prendre leurs victimes et les empêtrer. Laclos y dépeint aussi l'inégalité des sexes, les déficiences de l'éducation religieuse pour les jeunes filles, l'hypocrisie et la corruption d'une société frivole dominée par les apparences, et l'inefficacité de la justice. En plus, il est aussi question d'une inégalité sociale remarquable. Le peuple n'est représenté que par la présence de quelques servants, mais il est évident qu'ils sont les victimes faciles et les complices des manigances des seigneurs: c'est le cas d'Azolan, le chasseur de Valmont, de Victoire, femme de chambre de Merteuil, et de Julie, la servante de Tourvel qui est forcée de donner des informations sur la Présidente au Vicomte. On ne trouve pas, chez ces domestiques des Liaisons dangereuses, les vertus du peuple couramment célébrées par contraste avec la corruption de l'aristocratie comme il arrive dans le drame bourgeois de Diderot ou dans La nouvelle Héloïse de Rousseau.

Dans les années 1980 en Angleterre, la grande polémique à propos de la création d'une poll tax (une taxe fixe que tous les adultes résidents devaient payer) dans le Royaume-Uni par le gouvernement de Margaret Thatcher commence justement en 1985, l'année où Les liaisons dangereuses de Christopher Hampton ont été représentées pour la première fois.

La lecture politisée suggérée par la phrase en exergue des Liaisons dangereuses (1985) que nous avons mentionnée ("Comme devant tant d'œuvres de notre temps - pas seulement littéraires - le lecteur des Liaisons eût pu dire: 'Ça ne peut pas durer ainsi.") se confirme justement dans le dénouement, qui n'aurait évidemment pas pu exister à l'époque de Laclos. Comme nous l'avons déjà dit, au dernier tableau de la pièce, mesdames de Merteuil, de Volanges et de Rosemonde jouent aux cartes chez la première - le lecteur se rappellera le premier tableau du premier acte de la pièce où la Marquise jouait au piquet avec $\mathrm{M}^{\mathrm{me}}$ de Volanges. Elles parlent du destin de Tourvel, de Valmont, de 
Cécile et de Danceny. Quoique $\mathrm{M}^{\mathrm{me}}$ de Rosemonde cite "a very strange letter from Danceny" (LLD-H, p.100) qu'elle a reçue, le lecteur n'est pas sûr si la vieille dame connaît tous les faits et vilenies de la Marquise. En contraste avec la tristesse de ses amies, Merteuil entend la conversation impassiblement ("Merteuil listens, her practised expressionlessness intact, except for the glitter of satisfaction in her eyes", LLD-H, p. 99). Elle semble émerger indemne des événements tragiques et, dans la dernière réplique de la pièce, elle réfléchit sur les effets bienfaisants du temps et déclare son optimisme par rapport à l'avenir:

This has been a terrible few weeks. But time La Marquise est victime de l'ironie de l'auteur qui a le dernier mot: la didascalie finale évoque de façon explicite la dimension politique de sa lecture suggérée déjà par passes so quickly. A new year tomorrow and more than half-way through the eighties already. I used to be afraid of growing old, but now I trust in God and accept. I dare say we would not be wrong to look forward to whatever the nineties may bring. Meanwhile, I suggest our best course is to continue the game. (LLD-H, p. 101)

Après ces mots de Merteuil, une fugace atmosphère de sérénité domine la scène, les femmes reprennent leur jeu, mais alors: "Very slowly, the lights fade; but just before they vanish, there appears on the black wall, fleeting but sharp, the unmistakable silhouette of the guillotine" (LLD-H, p. 101).

Cette projection de l'image de la guillotine apparaît dans le scénario publié où une deuxième note de l'auteur - "Author's Note" - fait la remarque suivante: "What is published here is the text of the play as it stood on the first day of rehearsal; and the various minor cuts and abrasions (and improvements) sustained and effected during rehearsals are therefore not included“ (LLD-H, p. 6). En fait, peu de temps après la première de la pièce à Londres la silhouette de la guillotine a été remplacée par la projection de l'étendard bleu, blanc et rouge de la Révolution (Carson, 1991).

Ni l'image de la guillotine ni l'étendard de la Révolution ont été montrés au dénouement dans la version de la pièce présentée à Paris où la punition de Merteuil revient à ce qu'elle était dans le roman - les hués à l'Opéra, la petite vérole et la défiguration cachée sur scène sous un voile noir - ne semble pas donner à l'auteur la même opportunité d'évoquer la dimension politique de sa lecture du roman. Voyons ce 
que Hampton raconte lui-même (MACCUBIN, 1990, p. 85) à propos de ce changement par rapport aux premières représentations en Angleterre:

My translator said that this was such a famous book in France, he couldn't change the ending. So [...] we wrote a different last scene in which Mme de Rosemonde and Mme de Volanges discuss everything that has happened. A the back of the stage the mirror comes in slowly; as they get to the end of their conversation, they begin to talk about what happened to Merteuil, that she had been booed at the opera and that she'd got smallpox, and so on and so forth, as the actress playing Merteuil comes on in black with a veil and then stands in front of the mirror with her back to the audience and raises the veil. And the mirror is a distorting mirror, so that this awful face appears in the mirror. Rather effective. On the other hand, I prefer my ending.

Hampton semble sensible à la réception de sa pièce, au goût du public et à son adhésion au spectacle, mais même s'il attribue la raison de ce changement à l'attitude respectueuse du traducteur français vis-à-vis de l'œuvre de Laclos, la concession qu'il fait en dépit de ses préférences personnelles nous semble indiquer plutôt que l'image de la guillotine (ou de l'étendard tricolore), comme une nouvelle forme signifiante, était plutôt pertinente au spectacle montré au public anglais qui, un peu comme Merteuil - mais au XXe siècle -, était "more than half-way through the eighties already" en attendant "to whatever the nineties may bring" (LLD-H, p. 101).

La réception joue donc un rôle important dans la pratique de l'adaptation, les réactions du spectateur étant liées à sa culture, à son expérience individuelle. Les changements opérés dans la relecture d'une œuvre littéraire peuvent ainsi répondre au besoin de véhiculer des idées nouvelles dans un contexte socio-historique différent (la Grande-Bretagne de Thatcher dans les années 1980), mais dont les caractéristiques légitiment la réinterprétation et la transformation de l'intrigue ainsi que de nouvelles interrogations posées sur le roman. Quand le dramaturge anglais termine sa pièce avec une didascalie évoquant l'image d'une guillotine (ou de l'étendard tricolore) il veut signaler que les jeux de l'aristocratie vont bientôt terminer avec la fin des années 1780. Merteuil ne sera pas vaincue par le repentir, par l'émotion ou par la faiblesse comme les autres personnages de la pièce, mais par les forces de l'Histoire.

Hampton choisit ainsi de privilégier une interprétation très liée au moment historique où il produit sa version théâtrale des Liaisons dangereuses. Sa vision nous semble plus 
optimiste que celle de Laclos: il croit à la possibilité d'un triomphe sur le despotisme, quand il suggère que l'univers égoïste, rationnel et insensible des libertins et de l'aristocratie est destiné à périr.

L'auteur anglais apporte au roman du $\mathrm{XVIII}^{\mathrm{e}}$ siècle les reflets de la contemporanéité, du moment historique en face auquel il prend position. II fait ainsi éclater les limites de la scène pour parler de son temps, de sa propre culture et pour construire une l'intrigue littérairement très riche aux personnages remarquables inspirés par ceux du roman de Laclos. L'échange de lettres étant transformé en dialogue tranchant, c'est par le truchement des jeux d'acteur révélés par les didascalies, que les personnages - devenus effectivement des acteurs sur scène - dévoilent leur condition de libertins.

\section{BIBLIOGRAPHIE}

BAUDELAIRE, Charles. "Sur Les Liaisons dangereuses". In: CEuvres complètes. Préface, présentation et notes de Marcel A. Ruff. Paris: Seuil, 1968.

CARSON, Kathryn. Les Liaisons dangereuses on Stage and Film. Literature/Film Quarterly. 19 (1), 1991.

DELON, Michel. P-A Choderlos de Laclos /Les Liaisons dangereuses. Paris: PUF, 1999.

FIERRO, Danubio Torres. "Nova edição de 'As Relações Perigosas' permite revisar obra após dois séculos". O Ostado de São Paulo. http://www.estadao.com.br/noticias/arteelazer,nova-edicao-de-as-relacoes-perigosaspermite-revisar-obra-apos-dois-seculos,948064,0.htm. Sur l'Internet. 19/10/2012.

GRASSIN, Sophie. Doc Gynéco et Mr Bruno. Portrait. L'Express. fr, 3décembre1998.<http://www.lexpress.fr/mag/arts/dossier/rap/dossier.asp?ida=426897>. Sur l'Internet, 12/01/99.

GROSS, Robert (ed.). Christopher Hampton. A Casebook. New York \& London: Garland Publishing, "Coll. Casebooks on Modern Dramatists", 1990.

HAMMER, Stephanie Barbé. "Romanticism and reaction: Hampton's Transformation of Les Liaisons dangereuses. "In: GROSS, Robert (ed.). Christopher Hampton. A Casebook. New York \& London: Garland Publishing, "Coll. Casebooks on Modern Dramatists”, 1990

HAMPTON, Christopher. Les Liaisons dangereuses. London: Faber and Faber, 1986. 


\section{Literatura e Autoritarismo}

Identidade, memória e representações culturais

LACLOS. As Relações Perigosas. Tradução de Carlos Drummond de Andrade. Rio de Janeiro: Ediouro, "Universidade de Bolso", s/d.

MACCUBIN, Robert. Christopher Hampton interviewed by Robert Maccubin. Eighteenth Century Life. Volume 14, $\mathrm{n}^{\circ}$ 2, May 1990.

MACHADO, Maristela Gonçalves Sousa. Théâtre et libertinage dans Les Liaisons dangereuses : du roman à l'écran. Tese de doutorado em Literaturas francesa e francófonas. Instituto de Letras - Universidade Federal do Rio Grande do Sul, Porto Alegre. 2005.

POMEAU, René. Introduction. IN: LACLOS. Les Liaisons dangereuses. Établissement de l'édition, Introduction, notes et bibliographie par René Pomeau. Paris: Flammarion, "GF", 1981.

VERSINI, Laurent. "Les Liaisons dangereuses à la scène et à l'écran". In: Cent ans de littérature française 1850-1950: mélanges offerts à M. le Professeur Jacques Robichez. Paris: SEDES, 1987.

VERSINI, Laurent. "Des Liaisons dangereuses aux liaisons farceuses" In: Travaux de littérature. Vol. 6. Paris: Adirel/Klincksieck, 1993. 